\title{
Review of Traffic Safety Situation Based on Analysis
}

\author{
Muhammad Asim Azim ${ }^{1, \text { a }}$, Shamim Akhtar ${ }^{2, b}$, ZHAI-Fengwen ${ }^{3, c}$ \\ and WANG Yangping, $\mathrm{d}$ \\ ${ }^{1}$ School of Electronics and Information Engineering, Lanzhou Jiaotong \\ University, \\ Lanzhou 730070, China \\ $a_{*}$ gis.edu_lzjtu@yahoo.com
}

\begin{abstract}
Karachi is consider as foremost city of Pakistan regarding population and has huge contribution in entire economy, as well as has an attractive geographical position. But unfortunately entire city is facing immense causalities just because of bad road traffic safety system. The main purpose of this study is to review present safety situation, significance \& importance of feature study, study area causalities ratio than other major cities of Sindh province based on analysis-results, to highlight main safety issues and contributory factor.

Keywords: Review-Safety Situation, Analysis-Causalities ratio, highlight-Main issues, and Contributory factors.
\end{abstract}

\section{Introduction}

Globally, cost for road causalities is associated with trauma admission, of all health care centers putting enormous burden on low income and less resources countries. Unfettered transportation system and poor safety system have major roles to enhance economy burden. Road traffic accident is going to become the most cause of deaths because every year 1.2 million peoples are dying \& 50 million gets injuries just because of road accidents [1]. With regards to World Bank statistical report the road victims will boost up during year 2000 to 2020 $[2,3]$. Pakistan is suffering almost one hundred billion rupees every year, in maintenance side due to lack of road traffic safety. Generally the income resources of Pakistan's people are very low, almost 70 percent people of Pakistan are earning \$2 per day and most of the families are depended on one bread winner. Pakistan's population is approximately 170 million according to 2011 Population census whereas Karachi is considered as a mega city with approximately 23.5 million of population. Karachi is a very important city of Pakistan but due to poor economy and less resources, it is very difficult to control contributory factors threats. So some emergency level initiatives are required to face the challenges in reviewing actual safety situation based on analysis results. If we have a discussion about safety situation of Pakistan then Karachi's roads safety situation is 
competitively good than other major cities of Pakistan. But as well as this situation is not very comfortable if we analyze present safety situation with the major cities of developed countries. Therefore we divided manuscript into 5 sections to review actual situation; in the first section we give little introduction about feature and importance of our study area, in second section we analyze causalities ratio in Karachi with different cities of Sindh province, in third section we discuss first initial results of Karachi's RTI surveillance project, in forth section we analyze contributory factors, and in the section five we review about main safety issues.

\section{Significance \& Importance of Study Area}

Karachi is still considered as a metropolitan of Pakistan and consists of huge populated city of Pakistan, and now the capital of Sindh province and before it used to be the capital of Pakistan from 1947 to 1958. Complete city spread over 3,527 sq. kilo meters. Fundamentally it is divided into eighteen towns, 6 cantonment boards and a few agencies. It was estimated by population census in 2013 that Karachi has an estimated population of 23.5 million people and the 3rd largest city in the world by population. A substantial part of Sindh's gross domestic product (GDP) is attributed to Karachi (the GDP of Sindh as a percentage of Pakistan's total GDP has traditionally hovered around 28\%-30\%; and most of the foreign multinational corporations are operating in Pakistan but mostly they all have their headquarters in Karachi. But it is not considered as a rapidly growing cities in the world, and faces so many problems that are central to many developing metropolises, including road traffic safety. It is a vital city and located in the south of the country, on the coast of the Arabian Sea. It's geographic coordinates are $24^{\circ} 51^{\prime} \mathrm{N} 67^{\circ} 02 \mathrm{E}$. Karachi has been de-merged into its 5 original constituent districts namely Karachi East, Karachi West, Karachi Central, Karachi South and Malir. There are also six military cantonments which are administered by the Military.

\section{Analysis Causalities Ratio In Karachi With Different Cities Of Sindh Province}

Karachi is the capital of Sindh province and consider as metropolitan city (of Pakistan) faces high numbers of causalities. It is estimated by SRDO (Social Research and Development Organization) in 2012 that Karachi is facing high number of causalities comparatively other major cities of Sindh province. And "Hyderabad" is the second city having more road causalities after Karachi [2]. Meanwhile Both cities have high numbers of causalities than other major cities in Sindh province; like Larkana, Mirpur Khas, Sukkar, Thatta , as shows in figure.1 


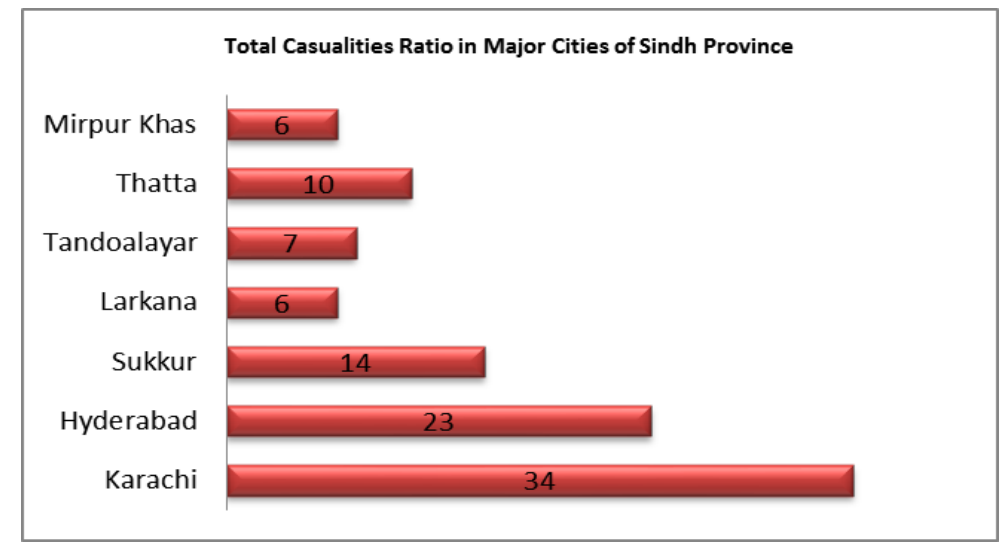

Figure.1: Causalities ratio in major cities of Sindh Province

\section{Initial results of Karachi’s RTI surveillance project}

From September 2006 to September 2009, it is estimated that there are two most common road users to be affected by road traffic accidents; riders and pillion of two wheelers vehicle and pedestrians. But the majority of fatal victims are pedestrians. It is estimated that only seven percent motorcyclists where found to be wearing helmets at accident time. And most common accident cause was rear-ending collision. Almost sixty nine percent victims brought by public transportation and twenty two percent brought by private ambulances. It is projected that ninety two percent victims died in government hospitals of the total fatal victims. Most accident victims got external wounds, such as lacerations, abrasions, etc. Overall reported outcomes confirmed that injury patterns in main city (Karachi, Pakistan) are normally similar to that reported others LMICs. However, there are a small number of differences in overall incidence and mortality rate. It is estimated during six years that there have a large number of male victims and larger proportion of victims being two wheeler vehicles [4].

\section{Last Five Years Contributory Factors}

Subsequent to confirmation contributory factors in Karachi, Pakistan, we examined some published reports related to actual numbers of causalities and causes. It is estimated that during last decade there is no big achievements to impede high threats in entire safety system. In favor of identification contributory factors, we consider last five years published reports by RTIR\&PC (2009-13). These reports clearly mentioned that injurious actions are one of major reason to enhance entire safety threat [5]. In other words without education awareness it becomes too difficult to control and hold back. Acknowledgements of all problems appreciate those new policies associated issues be able to make better safety altitude [6,7]. Injudicious actions are a main factor, have approximately 
45-47\% influences at entire safety system but we can’t avoid other important issues, as show in fig. 2 .

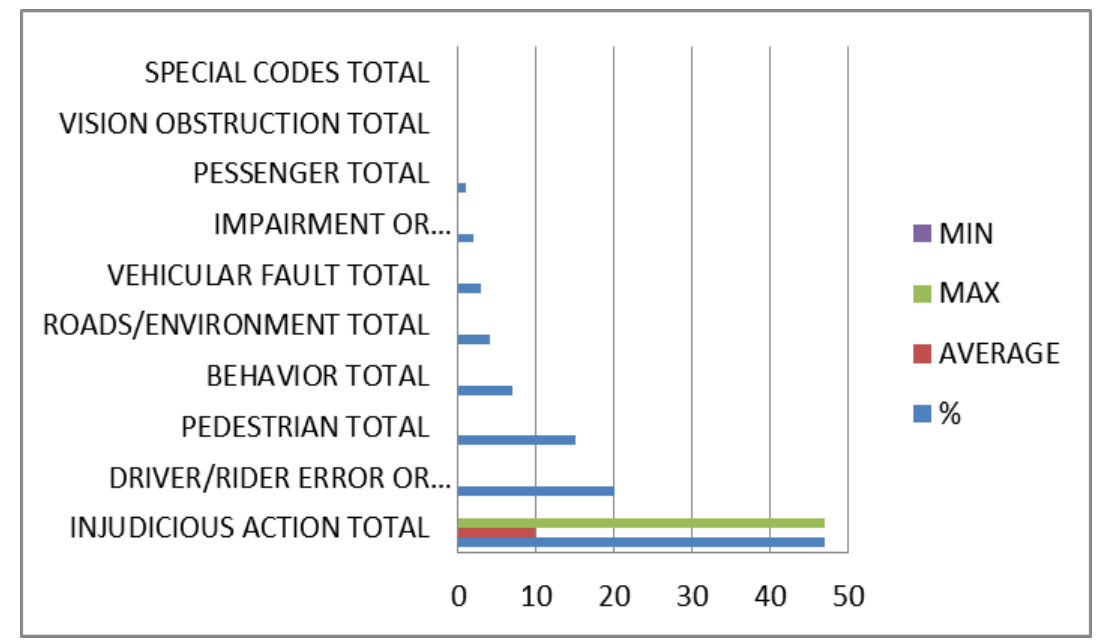

Figure 2: Last 5-Years Contributory Factors

\section{Karachi-Safety Issues}

There are total main eight safety issues are identified by safety authorities and stakeholders. These below issues are major and considerable issues to improve safety culture in Karachi, Pakistan.

\subsection{Road Conditions}

Karachi road conditions are not very well. This condition has become serious and causes road traffic accidents and extensive damage to vehicles. Many accidents happened just because of bad road conditions. There are a lot of other reasons involved in the damaging of the roads. There have poor drainage system that is also responsible for worsening conditions of the roads. In fact present situation is really bad and painful for public who need to use these roads to move from one place to another place. In reality whole situation become more worst if provincial government doesn't take any action on emergency bases. Because it is a right of every citizen in Karachi to have better roads in utilizes. The entire transport structure is badly affected due to the poor conditions of the roads. The concerned authorities are requested to take needed action in regarding roads improvements.

\subsection{Institutional Issue:-}

$\checkmark \quad$ Due to lack of resources there is no proper system to sustain coordination and collaboration among traffic safety stakeholders and identified institutional problems. 
$\checkmark$ Currently, there is no formal communication and data sharing mechanism among safety agencies.

$\checkmark \quad$ Put burden on financial by insufficient allocation of human resources on road safety program.

$\checkmark \quad$ No extra funding to start road safety champions for road safety improvements on highway.

$\checkmark \quad$ Don't have experts to train traffic operation engineers and auditors.

$\checkmark \quad$ Lack of training opportunities to improve road safety professional skill.

$\checkmark$ Reliance on high fee international Consultants which restricts the opportunities for safety improvements.

\subsection{Road Users Issues}

$\checkmark \quad$ Pedestrians often would like J-waking due to lack of safety awareness and specially most of children are often affected by lack of awareness. The existing rules have no punishments for their unsafe practices so safety legislation required to draw some polices for pedestrian safety.

$\checkmark \quad$ Helmet is very necessary for motorcyclist but ratio of helmet using is very low. Major factor of low ratio is weather and restrictions. Safety legislation must define some strict rules for motorcyclist safety.

$\checkmark \quad$ Same like motorist generally don't like wear seat belt. Because there is no check and balance and not properly defined safety legislation, specially related to punishment. Before due to unawareness there had some relaxation but now traffic police started campaigns for awareness related to seat belt. And most of the old vehicles are not equipped with seat belts.

$\checkmark \quad$ Most of pedestrians do not pay any attention to make them visible on road under dark condition.

$\checkmark \quad$ Use of mobile phones during car and/or motor-biking driving is becoming normal day by day.

$\checkmark \quad$ Motorbike front light is mandatory to keep but lack of public awareness and traffic police is not conscious of this safety measure.

\subsection{Public Transportations Issues}

$\checkmark \quad$ Public transportation condition is too bad, a roughly estimated, up to twenty years old vehicles are on our roads.

$\checkmark$ Deficiency of public transpiration is reasoning of overloading in public vehicles.

$\checkmark$ Mostly uneducated persons are drivers of public vehicles don't have awareness how to deal with public and maintain vehicles.

\subsection{Road Infrastructure Issues}

$\checkmark$ Most of infrastructure companies doesn’t follow safety design standard during roads construction. 
$\checkmark \quad$ Old infrastructure equipment are used during constrictions.

$\checkmark \quad$ There is no rule defined by legislations on U-Turns and most of U-Turns made by business owners for personal advantage are causing road accidents.

$\checkmark \quad$ Underpass blocked during rain just because of bad poor designing.

$\checkmark$ Some roads are congested due to unauthorized parking spaces are causing road crashes.

$\checkmark \quad$ There is not a well-defined QC/AQ mechanism in safety designing.

\subsection{Vehicle Safety Issues}

7. Illegal fabrication of public vehicles to increase load for personal advantage are cause road accidents and road damage.

8. Overloading is severe threat for road safety and disturbing flow by slow moving on roads.

9. Illegal make use of high intensity lights by vehicles drivers causing road accidents during night time.

10. New vehicles are equipped by fog lights but some drivers have not proper knowledge that how to use and avoid crashes.

11. There is no proper vehicle safety inspection by road safety authorities to save public lives and control vehicles damage.

\subsection{Legislation/Regulation Issues}

$\checkmark \quad$ Motor Vehicle Ordinance and Highway Ordinance have same agenda for safety improvement but need to be combined in one document.

$\checkmark \quad$ Many road accidents happened by long cargo container high speed. There is no legislation to check fabrication and inspection.

$\checkmark$ Most of vehicles drivers are involved in injudicious actions. But victim's relatives have no awareness about legal rights and compensation. By safety authorities needs to start campaign for victims' rights.

$\checkmark \quad$ Present fine structure is not enough to change behavior especially for those vehicles driver whose are involved in dangerous adventures. Traffic police must take some serious actions to impede them. And need to increase fine and involve some strict punishments.

$\checkmark$ Most of insurance companies are fake because there is no accountability system. So safety authorizes must take serious actions to prevent "victims" rights to encourage safety culture.

\subsection{Hospital/EMS Issues}

$\checkmark \quad$ Working trauma centers are not enough to fulfill present requirements.

$\checkmark \quad$ There is no government based ambulance service. Only EDHI and AMAN ambulance service has major roles to receive crash victims or medical related victims. And some other NGOs are providing transportation facilities but these ambulances are not fully equipped.

$\checkmark \quad$ Lack of professionals to provide basic life support. 
$\checkmark \quad$ Due to roads congestion and rush hours sometimes serious injured victims dies because currently safety authorizes have not enough capacity of air ambulances/transportation to facilitate serious injured victims.

\section{Discussion}

In the past, a lot of countermeasures were considered to reduce road safety threats, like: deaths and injuries. However, the recent research shows that there are still a lot of factors involved enhancing road safety threats. But in reality identification of problems and emergency based initiatives helps out safety related departments to control anxiety situation. Meanwhile there are still a lot of necessary things to do to emphasize better safety . No doubt, road traffic safety is a global issue but it is a significant issue for developing countries because almost $90 \%$ occurrences happened in developing countries and as one of the most serious problem the society is facing today[8-11]. This manuscript draws our attention to serious issues for all road users groups including all the vulnerable ones. The first complete report about safety issues lunched by Road Traffic Injury and Prevention Centre (Karachi, Pakistan) with the help out of World Bank and World Health Organization(WHO) in 2002. Main focus of this document is to address the issues and stress the urgent need for actions to prevent road safety culture, and open up the eyes of all safety related departments in region of Sindh. Most of Causalities are reported to the police and trauma centers of Karachi belongs to the vulnerable road user groups, because approximately 45 to 47 percent causalities just happened by vulnerable actions. Apart from that other aspect in road safety there are some other factors such as Enforcement problems, Rough driving, Overloading, Vehicle maintenance, Bad road conditions, Human contributory factors, communication barrier, EMS issues as well.

\section{Conclusions}

The main objective of this research is to highlight present situation based on causalities ratio, significance of study area, contributory factors and main safety issues. Karachi is the most important city in Pakistan and considered as main participant in entire country economy. But still there have no proper road traffic safety system; just because of this reason Karachi City economy has badly affected. In this review paper we try to highlight some present considerable issues by analyzing results. In short, an injudicious action is consider as a one big reason with $45 \%$ to $47 \%$ influences in entire traffic safety, but we also cannot avoid other important issues related to road traffic safety. If safety agencies or departments prepare policy draft after acknowledged actual situation step by step in priority bases then situation can become in control and can be better. No doubt, Behavioral change programs and enforcement Importance have a massive role in safety improvements regarding educational/informative awareness road safety programs are aimed towards providing data to road users regarding secure practices to take on to engage in traffic safely. The main problem is pinpointing the actual situation, 
identification of incident black spots, rapidity incident locations and as well as the incident causes. Because current recorded information procedure is not enough to find present situation, and as well as for further analysis.

\section{References}

[1] Razzak, J.A. and L. Laflamme, Limitations of secondary data sets for road traffic injury epidemiology: a study from Karachi, Pakistan. Pre hospital Emergency Care, 2005. 9(3): p.355-360.

[2] United Nations. United Nations Road Safety Collaboration. Global plan for the decade of action for road safety 2011-2020 [Internet]. 2011. Available from: http://www.who.int/roadsafety/decade_of_action/plan/plan_english.pdf

[3] Peden MM, Scurfield R, Sleet D, Mohan D, Hyder AA, Jarawan E, et al. World report on road traffic injury prevention. Geneva:WHO; 2004.

[4] Sheraz., E.B.A.L.A.E.K., Road Accident on Sindh,2012; Situation Analysis. Social Research and Development Organization, SRDO., 2012. Traffic News \& Incident Reporting 2012 by SRDO: p. p.6.

[5] Shamim, S., et al., Initial results of Pakistan's first road traffic injury surveillance project. International journal of injury control and safety promotion, 2011. 18(3): p. 213-217.

[6] Ghaffar A, Hyder AA, Masud TI. The burden of road traffic injuries in developing countries: the 1st National Injury Survey of Pakistan. Public Health 2004; 118:211-7

[7] World Health Organization, violence and injury prevention and disability (VIP). Road traffic injuries. 10 facts on global road safety. Geneva: WHO; 2011.

[8] Ghaffar A, Hyder AA, Bishai D, Morrow RH. Interventions for control of road traffic injuries: review of effectiveness literature. J Pak Med Assoc 2002; 52:69-73.

[9] Ministry of Health Govt. of Sindh, P.S.B.A.F., ROAD TRAFFIC INJURY RESEARCH \& PREVENTION CENTRE SIX YEARS PERFORMANCE REPORT WITH STATISTICAL REPORT 2011-2013. Department of Neurosurgery, Jinnah Postgraduate Medical Centre, Rafiqui Shaheed Road, karachi-75510, Pakistan.

[10] Global Plan for the Decade of Action for Road Safety, 2011“C2020. Geneva, World Health Organization,

2011(www.who.int/roadsafety/decade_of_action/plan/plan_english.pdf,accessed 24january2013)

[11] Razzak JA, Shamim MS, Mehmood A, Hussain SA, Ali MS, Jooma R. A successful model of road traffic injury surveillance in a developing country: process and lessons learnt. BMC Public Health 12:357. 